Bond University

Research Repository

\title{
Tapering practices of New Zealand's elite raw powerlifters
}

Pritchard, Hayden J.; Tod, David A.; Barnes, Matthew J.; Keogh, Justin W.; Mcguigan, Michael R.

Published in:

Journal of Strength and Conditioning Research

DOI:

10.1519/JSC.0000000000001292

Link to output in Bond University research repository.

Recommended citation(APA):

Pritchard, H. J., Tod, D. A., Barnes, M. J., Keogh, J. W., \& Mcguigan, M. R. (2016). Tapering practices of New Zealand's elite raw powerlifters. Journal of Strength and Conditioning Research, 30(7), 1796-1804.

https://doi.org/10.1519/JSC.0000000000001292

\section{General rights}

Copyright and moral rights for the publications made accessible in the public portal are retained by the authors and/or other copyright owners and it is a condition of accessing publications that users recognise and abide by the legal requirements associated with these rights.

For more information, or if you believe that this document breaches copyright, please contact the Bond University research repository coordinator. 


\section{Tapering practices of New Zealand's elite raw powerlifters}

Tapering practices of powerlifters

Hayden J Pritchard - ${ }^{1}$ Lecturer in the Department of Exercise \& Sport Science, Faculty of Health Science at the Universal College of Learning, Palmerston North, New Zealand; and, ${ }^{2} \mathrm{PhD}$ student at the Sports Performance Research Institute New Zealand, Auckland University of Technology, Auckland, New Zealand.

Dr David A Tod - Senior Lecturer in the School of Sport and Exercise Sciences at Liverpool John Moores University, Liverpool, United Kingdom.

Dr Matthew J Barnes - Lecturer in the School of Sport and Exercise at Massey University, Palmerston North, New Zealand.

A/Prof. Justin W Keogh - Associate Professor in the: ${ }^{1}$ Faculty of Health Sciences and Medicine at Bond University, Queensland, Australia; 'Sports Performance Research Institute New Zealand, Auckland University of Technology, Auckland, New Zealand; and, ${ }^{3}$ Cluster for Health Improvement, Faculty of Science, Health, Education and Engineering, University of the Sunshine Coast, Queensland, Australia.

Prof. Michael R McGuigan - Professor at the Sports Performance Research Institute New Zealand, Auckland University of Technology, Auckland, New Zealand.

Corresponding Author:

Hayden Pritchard

UCOL Exercise \& Sport Science

Private Bag 11022

Palmerston North 4442

New Zealand

Phone: (06) 9527001 ext: 70657

Email: h.pritchard@ucol.ac.nz 


\section{ABSTRACT}

The major aim of this study was to determine tapering strategies of elite powerlifters. Eleven New Zealand powerlifters ( $28.4 \pm 7.0$ years, best Wilks score of $431.9 \pm 43.9$ points) classified as elite were interviewed, using semi-structured interviews, about their tapering strategies. Interviews were transcribed verbatim and content analyzed. Total training volume peaked $5.2 \pm 1.7$ weeks from competition while average training intensity (of $1 \mathrm{RM}$ ) peaked $1.9 \pm 0.8$ weeks from competition. During tapering volume was reduced by $58.9 \pm$ $8.4 \%$ while intensity was maintained (or slightly reduced) and the final weight training session was performed $3.7 \pm 1.6$ days out from competition. Participants generally stated that tapering was performed to achieve full recovery; that accessory work was removed around two weeks out from competition; and, deadlifting takes longer to recover from than other lifts. Typically participants stated that trial and error, and changes based on 'feel' were the sources of tapering strategies; equipment used and movements performed during tapering are the same as in competition; nutrition was manipulated during the taper (for weight cutting and/or performance aims); and, poor tapering occurred when too long (one week or more) was taken off training. These results suggest that athletes may benefit from continuing to strength train prior to important events with reduced volume and maintained intensity. Only exercises that directly assist sports performance should remain in the strength program during tapering, to assist with reductions in fatigue while maintaining/improving strength expression and performance.

Key words: peaking, strength, training 


\section{INTRODUCTION}

Powerlifting is a strength sport that includes three barbell based lifts: the squat, bench press and deadlift. The major governing body is the International Powerlifting Federation (IPF) who sanctions competitions in accordance with the World Anti-Doping Agency (WADA) code. The objective of the sport is to lift the largest combined weight across the three lifts during competition (10). Swinton et al. (23) investigated the training practices of elite British powerlifters and found nearly all athletes implemented some kind of periodization into their training. Like most athletes, powerlifters will target specific competitions during the competitive year for maximal performance, so this periodized training must culminate with an effective taper to ensure peak performance.

Tapering is the last stage in a well-planned training program and has the ability to determine the success or failure of the training period prior to competition (7). Mujika and Padilla (15) have described tapering as a nonlinear reduction in the training load over a period of time which reduces the stress, or fatigue, of training while improving fitness to achieve optimal performance at a specific time. Tapering will usually result in a performance improvement of around 3\% (range of 0.5-6\%), which in some sports could be the difference between winning and not making the podium $(13,20)$. It has been shown that a taper of less than four weeks with volume reductions of around 50-90\%, maintaining or slightly increasing training intensity, whilst maintaining training frequency is most effective for experienced endurance and anaerobic athletes (29). An athlete who is fatigued requires a longer tapering duration and greater training load reductions (24). However, there is currently limited specific tapering research examining powerlifters and other strength sports.

Research that has investigated tapering for strength has shown that when intensity is maintained or slightly increased during the taper greater performance improvements may occur $(5,6,11)$. In these studies training volume reductions of $30-70 \%$ occurred through reduced training frequency or training session volume over a period of one to four weeks (19). It may be hypothesized that a taper maintaining or increasing training intensity, while decreasing training volume, is an effective strategy for enhancing maximal strength. However, 
it is unknown if the strategies currently employed by strength athletes agree with the current literature. If athletes' practices are similar to research supported recommendations this would provide further confirmation to coaches and practitioners that these strategies should be adopted. It is also possible that athletes' may employ strategies that differ from the literature yet are successful, if so this would require further investigation.

The major aims of this study were to gain some insight into the current tapering strategies of elite powerlifters, why such strategies are employed, how these strategies were developed and how tapering might differ for each lift. Insights from this study will provide useful information on peaking strategies which can be applied by strength coaches and athletes. Results from this study may also help athletes from other sports optimize their strength, and competitive sports performance - especially those who are well strength-trained.

\section{METHODS}

\section{Experimental Approach to the Problem}

Semi-structured interviews with elite raw IPF powerlifters in New Zealand were used to collect data needed to achieve the aim of the study. Semi-structured interviews ensured information surrounding people's experiences of tapering could be obtained, which may be missed with alternative methods (such as surveys) that predetermine the type of data collected. Previous research has revealed that semi-structured interviews and surveys can capture elite athletes' and coaches' experiences and practices in ways that allow others to learn from their experiences $(25,30,31)$. To ensure current elite powerlifters were recruited for the study, the criteria for an invitation to participate was the achievement of a raw competition total (combination of athletes' best squat, bench and deadlift) classified as 'elite' by the New Zealand Powerlifting Federation's (NZPF) raw lifting qualification standards within the 12 months prior to the commencement of this investigation (see Table 1 for NZPF standards). Competitions run by the NZPF are sanctioned by the IPF and comply with the WADA code. No athletes interviewed had failed drug tests, ensuring results are applicable to drug-free athletes. As the participant pool was limited, the aim was to have as many lifters as possible 
participate in the study. Our initial target was to get at least 10 participants, then as many additional participants above this number as possible - a total of 11 lifters from various weight classes completed the study. Sample sizes in studies using qualitative methods are often of a similar size because of the depth of data collected from each individual (25). In such studies the focus is on understanding a few individuals in depth rather than gaining a superficial understanding of many people (25). Nevertheless, given that there were 16 individuals who may be classified as New Zealand elite lifters at the time of data collection, our sample represents $70 \%$ of the total population. Raw (or classic) powerlifting was chosen because it is performed without the aid of specialized supportive equipment (10) and so findings can be considered more appropriate to athletes from other sports.

INSERT TABLE 1 ABOUT HERE

To ensure we obtained relevant data from our semi-structured interviews we obtained answers to a specific set of guiding interview questions, however, these questions allowed some freedom for participants to discuss related information and experiences they felt relevant to their tapering practices. Questions asked were designed to ensure we collected data that would answer our research questions - determining general (and lift specific) tapering strategies, how such strategies were chosen and the reasons for strategies employed. Following pilot testing on two international powerlifters, the questions were refined before being used for data collection.

\section{Subjects}

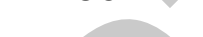


the study due to time commitments or other, undisclosed, reasons. Prior to involvement in the study, all participants received written explanations of the study's risk and benefits and provided informed consent.

The participants (eight men, three women) had a mean age of $28.4 \pm 7.0$ years, best competition total (within 12 months of the study) of $615.5 \pm 158.1 \mathrm{~kg}$, best Wilks score (within 12 months of the study) of $431.9 \pm 43.9$ points, competition bodyweight of $91.0 \pm 27.4 \mathrm{~kg}$ and trained $4.8 \pm 0.5$ days per week. The eight men had a mean age of $26.5 \pm 5.4$ years, best competition total of $693.8 \pm 97.8 \mathrm{~kg}$, best Wilks score of $450.3 \pm 31.9$ points and competition bodyweight of $94.9 \pm 29.9 \mathrm{~kg}$. The three women had a mean age of $28.4 \pm 7.0$ years, best competition total of $406.7 \pm 39.9 \mathrm{~kg}$, best Wilks score of $431.9 \pm 43.9$ points and competition bodyweight of $91.0 \pm 27.4 \mathrm{~kg}$. The Wilks score is a bodyweight adjusted scoring system which allows comparisons in weight lifted across weight classes (10). All participants had previously achieved medals at IPF International Competitions (minimum of Oceania Championships). Nine participants said they were self-coached, and two said they were semi-coached (i.e. a coach had some input but was not in full control of their training).

\section{Procedures}

All participants were initially approached via email. The email outlined the study's aims, risks and benefits, and invited athletes to participate in the study. It also gave participants information regarding the types of questions that would be asked, and a chance to ask any questions of the researcher prior to their involvement. Giving participants such background information means that they have a chance to reflect on their practices and improves the value of data collected (25). After providing informed consent, interviews were arranged; eight interviews occurred via telephone (or Skype voice calls) and three face to face. Interviews took 30-60 minutes to complete and were recorded using a Sony integrated circuit recorder (Sony IC Recorder ICDAX412F, Sony Corporation, Tokyo, Japan). Throughout the interview the researcher also took notes on participants' responses. Following the interview, recordings were transcribed verbatim. Telephone interviews allowed data to be collected from all suitable participants without the issue of location. It has been shown that telephone interviews produce comparable quality and scope of data as face to face interviews while improving participation rates (22). 
Aside from initial demographic and performance based questions, all guiding interview questions were based around participants' training practices and in particular how they taper, or peak in the final few weeks of training before an important competition. Many of the questions asked were open ended which gave participants the freedom to discuss what they thought was relevant to their tapering practices (17), while some questions allowed specific quantitative information on their tapering strategies to be determined (such as training volume, intensity and frequency etc.). The questions asked during interviews are shown in Table 2.

INSERT TABLE 2 ABOUT HERE

\section{Statistical Analyses}

Where data were quantifiable (such as training volume, intensity and frequency etc.) descriptive results were calculated for the sample. T-tests were used to determine differences between each lift (paired, two tailed ttest). Significance was set at $P \leq 0.05$. Where significance was found, effect sizes (ES) were also calculated (3). Hopkins' scale for determining the magnitude of ES was then used to describe magnitudes (8); these were: trivial 0-0.2, small 0.2-0.6, moderate 0.6-1.2, large 1.2-2.0 and very large $>2.0$. Analysis was performed using computer software (Excel; Microsoft Corporation, WA, USA).

Qualitative data was subjected to a thematic content analysis following Patton's (17) guidelines as used with previous data collected from a strength and conditioning context (25). A similar approach has also been used elsewhere within sport science literature $(21,26)$. Raw data units were arranged into common themes that address the research question. Specifically, the researchers read through the interview transcripts to find information related to the guiding interview questions and then summarized the information under labels that reflect the content of the theme. Themes addressed the overall research questions. Frequencies of specific themes were determined to help establish the number of people in this sample providing similar responses to interview questions. Themes were classified according to the representativeness shown, as performed by Tod et al. (25). Classifications were: general, themes applying to all or all but one participant; typical, themes 
applying to more than half the participants, but less than general; variant, themes applying to two or more participants, but less than typical.

\section{Validity and Reliability}

Three types of triangulation (2) were used to establish validity and reliability within this study. Firstly, participants across many weight classes and from many regions throughout New Zealand were recruited for the study and expressed similar themes, showing external validity in that similar themes emerged amongst these individuals. Secondly, member checking - providing participants with the researchers' interpretations of their data (2) - also occurred, ensuring that participants agreed that the results reflect their realities. Participants had both their own transcripts and interpretations of themes from all participants returned to them. No participant reported that we had misinterpreted or misrepresented them in their transcripts or our subsequent interpretations, with only minor wording corrections were required in two participants' transcripts. Thirdly, critical peer review - or analyst triangulation - occurred in that two other people (aside from the lead researcher) with expertise in differing areas evaluated the results and agreed that the themes expressed were representative of the data collected.

RESULTS

\section{Quantitative Data}

Participants generally followed a linear periodized approach with volume reducing and intensity increasing nearer to competition. Total training volume peaked $5.2 \pm 1.7$ weeks from competition while average training intensity (of $1 \mathrm{RM}$ ) peaked $1.9 \pm 0.8$ weeks from competition, with the heaviest individual session (where the highest intensities, \%1RM, are used) around $2.3 \pm 0.7$ weeks out from competition. As a competition approached the focus became predominately on competition lifts, with both squat and deadlift accessory work removed $2.0 \pm 0.7$ weeks from competition and bench press accessory work removed $1.9 \pm 0.7$ weeks from competition. Participants stated that their taper length was $2.4 \pm 0.9$ weeks out from competition. During this 
taper, participants stated that they maintained training intensity, however they estimated that volume was reduced by $58.9 \pm 8.4 \%$ during the taper. The final week was often a drastically reduced "deload" week with reductions in both volume and intensity, with training frequency reduced to $34.7 \pm 14.2 \%$ of usual training frequency and the final weight training session performed $3.7 \pm 1.6$ days out from competition. A tapering timeline for subjects is shown in Figure 1.

INSERT FIGURE 1 ABOUT HERE

Table 3 shows the final 'heavy' (>80\% of $1 \mathrm{RM}$ ) training session for each competition lift, as well as the final training session - regardless of weight - for each competition lift. Statistically significant differences were seen in that the final deadlift training session was performed further from competition than the relevant squat or bench press sessions (both moderate $E S=1.13$ and both $P=0.004$ ), the final heavy deadlift training session was performed further from competition than the relevant heavy squat (moderate $E S=0.85$ and $P=0.005$ ) or heavy bench press sessions (moderate $\mathrm{ES}=1.09$ and $\mathrm{P}=0.003$ ), and also, the final heavy bench press session was performed at a higher relative intensity than the final heavy deadlift session (small ES $=0.56$ and $\mathrm{P}=$ 0.033; this also approached significance when compared to the final heavy squat session, small ES $=0.40$ and $P$ $=0.057)$.

\section{Qualitative Data}

INSERT TABLE 3 ABOUT HERE

Table 4 presents the broad themes and subthemes from the interviews related to the research aims and their sample representativeness. Italicized quotes below illustrate participants' views and the number in brackets represents the percentage of participants who expressed that theme.

INSERT TABLE 4 ABOUT HERE 


\section{Tapering Differences between Lifts}

More recovery needed for the deadlift (90.9\%). Almost all participants commented that the deadlift required a longer recovery period before competition. Generally they felt that "it takes longer to recover, it's harder on the body" and "it will be the one I tend to be most sore from so I need an extra couple of days". As well as requiring the most days off, participants also felt this wasn't an issue because it "is probably not a movement I feel like I am going to forget or loose the groove for and I don't want to run the risk of taxing the body in any way" and that "you can go a couple of weeks without doing it and it's really not going to make much of a difference", indicating that the deadlift takes longer to recover from, while potentially requiring less frequent training.

Bench press \& squat tapering performed in a similar manner (45.5\%). Almost half of the participants followed similar tapering strategies in both their bench press and squat, such as "my last training session is usually squat and bench press, at my starting [competition opening] numbers", "I would work up to an opener... about a week out for a squat... [final heavy bench press session is] one week out at opener" and "I will do the bench press and the squat together [8 days out] and the deadlift will be on its own [two weeks out]". This approach is an extension on the fact that the deadlift takes longer to recover while the other two lifts do not seem to have the same fatiguing effects.

Bench press can stay heavier for longer (36.4\%). In contrast to the above, some participants felt that the bench press can stay heavier until closer to competition than the other lifts. Statements such as "I feel like it is a stronger lift and I can handle it a little closer to the comp... I can bench heavy and two days later bench heavy again" and "I do tend to keep that [bench press] quite heavy right up to close and keep that intense" showed that some participants appear to have little issues recovering from heavy bench press.

\section{Information sources for taper strategy}


Trial \& error (81.8\%). Generally participants had come up with their tapering strategies through their own failures in the past. It wasn't normally based on scientific information but "has come about by trial and error". They commented things such as they "guess it's just trial and error. It seems to be working, what I am doing" and others that over time they have "learned through all the competitions just how long it takes me to feel good again or how long my body needs to recover". This trial and error, or learning through past experience, was expressed by nearly all participants.

By 'feel' (63.6\%). Often lifters may have a set plan but they adjust it as needed. Some participants said after harder training than expected that "I feel like I need the rest to get myself ready" and others that "I have learnt how to read my body", and so adjust their taper accordingly. Through past experiences they have learnt how to adjust their taper based on how their body feels. They may make changes within a training session during the taper depending on how they feel or how previous training has gone, "purely on what I feel". This was a typical theme, the ability to "adjust the program leading up to the competition".

Previous coaches (36.4\%). Although at the time of the study only two participants had input from a coach, several had been previously coached and expressed that their original tapering strategies were formed then. Some were told "to rest for that time frame and that has worked for me when I have listened", others stated "he [the coach] would lead the way and how to taper and tell me when I needed to stop training and gave me a bit of a start to see how it feels to train different ways" and simply "it was my old coach in Perth - it was his logic". In essence coaches led initial tapering strategies which were then adjusted to what worked through trial and error.

Other lifters (18.2\%). There were a few participants who commented that "it was when I first came into powerlifting that was how everyone did it" and "it was because someone else told me to do it". Interestingly both of these lifters later changed their tapering strategy because what they were told worked didn't work well for them.

\section{Motives for tapering strategy}


To fully recover (90.9\%). Nearly all participants expressed the view that reducing training load during the taper was to allow for full recovery. Comments such as "resting up and getting my muscles back to 100\%", "you don't need that loading, you have done all the hard work it is more maintaining the weight you are lifting without doing overload training. So you recover more", "trying to achieve maximum recovery", "giving my body enough time to recover" and "I generally feel like giving my body a break and just let it recover". Clearly, recovery during the taper is a major motive in order to improving performance in competition.

Light competition movements for familiarity (45.5\%). Lifters typically expressed the need to continue to do the competition movements to keep them familiar. This was made clear through statements such as "I realized that I needed to keep working a little bit more into these meets so didn't feel as lethargic", "I have to do the light movement a few days out" and "still feeling like the movements are familiar and that I still know the groove for that particular exercise". Continuing to do competition movements appears to be imperative to keep the body and mind focused on the movements.

Time off heavy work makes them 'hungry' to lift (18.2\%). A couple of participants expressed that by avoiding heavy work they feel excited and 'hungry' to lift on competition day. One stated that "by the time Saturday comes I feel really good, I am hungry for the weight", while the other said "part of it is a mental thing, that amount of time is about how long it takes me to get really excited about an event and to lift again".

Taper focus is on the competition lifts

Accessory work is removed during the taper (90.9\%). During the final stages of training nearly all participants expressed that they remove the accessory, or non-competition, movements: "none of them [final heavy training sessions] have accessories in them", "I take it [accessory work] out two weeks out for all three lifts", "I remove all assistance work three weeks out" and "I drop all of them [assistance lifts]". One participant stated that they remove these lifts to ensure they can "go in and train the heavier weight [on competition lifts]", showing that this change is to allow the focus to be on the three competition lifts. 
Equipment is used on heavy sets as it would be in competition (81.8\%). Typically participants stated that they train like they will compete when lifting heavy sets during the taper. When asked if they alter equipment (wrist wraps, knee sleeves etc.) use, typical responses were "I use it all", "I try and keep everything similar to what I have on comp day" and "everything you are going to use in competition". The focus was on doing things like they will be done on competition day, as one participant put it, "practicing my routine".

\section{Other strategies employed during the taper}

Nutrition (72.7\%). Many participants undertook changes in their nutrition. Changes were made that they believed assisted recovery "I am tracking my food, I am trying to get a 40/30/30 [carbohydrate, protein, fat] split that seems to make me feel good and recover quicker"; improved performance "normally my training is at a maintenance level of calories and I will add in another $10 \%$ on top of that leading to a competition", "cutting out caffeine for a week - just to get maximum advantage come competition day", "always have a good carb feed that night and try get another one the night before competition"; helped lower bodyweight "I slowly fluctuate [bodyweight] down and then miss the odd meal that I don't feel like I need, like I will have a workout then just have a few eggs or something after it"; and, general health "I change my diet leading to a competition, I go $100 \%$ gluten free about six weeks from a competition, I'm a little bit gluten intolerant so I cut all gluten out of my diet".

Mobility Work - stretching, foam rolling, massage etc. (45.5\%). One theme expressed by participants was the use of mobility work for recovery. Some participants said "I would normally have a massage about 2-3 days out from competition", "I will do some [foam] rolling out or sometimes I go and get a massage. I do try to stretch out and [keep] mobile during that time", "I will do full a full on mobility session afterwards [light training week of competition], just to keep myself moving" and "I need the recovery time to let every muscle heal as much as possible, I do mobility stuff, stretching stuff during that week". Mobility work took various forms but with the same goal, to assist recovery and keep the body moving. 
Poor tapering experiences

Taking too much time off -1 week+ (54.5\%). A common theme was that when things had gone wrong, too much time had been spent away from training. This was expressed as participants said things such as "everyone just stopped training seven days out. So I did that for my first few comps and then after learning about my body a bit more I realized that I needed to keep working a little bit more into these meets so I didn't feel as lethargic.", "one week out I didn't lift at all. It was because someone else told me to do it and it didn't work.", "I just didn't train for the whole week before and I didn't like that" and "tapering too soon or backing off the weights too soon". These theme echoes the earlier theme of the need for keeping movements in for familiarity.

Over exertion too often prior to taper (36.4\%). Several participants expressed that tapering did not work when "I hit too many peaks through the build-up in nine weeks of training... there was no linear progression" or "a week later I had weight lifting nationals so doubling the two sports ruined my taper" and "whenever anything has gone wrong it is because I have pushed too hard on my last sessions". These participants tried to go too heavy too early and so broke the progression or fatigued themselves before meet day arrived, resulting in poor performances.

Cut too much weight (36.4\%). Cutting weight is often required for powerlifters to compete in lower weight categories, and it is also a cause of much stress and sometimes poor performances. Several participants stated things to that effect such as "I think one year it was because I was dropping weight", "everything went wrong because I had to cut too much weight" and "it is something else to think about as well, it is another stress to add to the equation. You are constantly thinking about weight all the time". Although not all participants who cut weight expressed this, a few did, it was particularly an issue when too much weight had to be cut, and one participant said they performed a "four week cut to get from $80 \mathrm{~kg}$ to $74 \mathrm{~kg}$ - it was horrible".

\section{DISCUSSION}


The current study is the first to document the tapering strategies employed by elite powerlifters. Participants reduced training volume but kept intensity relatively high, with a competition lift focus during the taper. It was generally believed by the participants that the deadlift requires a longer recovery, and the focus of a taper is on achieving full recovery with accessory work being removed. Typically participants stated that (a) trial and error and changes based on 'feel' were the information sources of tapering strategies chosen, (b) during the taper lighter competition movements are kept to maintain movement familiarity, (c) equipment used during the taper is the same as in competition, (d) nutrition is manipulated during the taper and (e) poor tapering occurs when too long (one week or more) is taken off training.

The final training session was performed at $3.7 \pm 1.6$ days out from competition, with eight of the eleven participants performing their final session three days out from competition, this is similar to previous research focused on other populations $(27,28)$. Weiss et al. $(27,28)$ have demonstrated on two occasions that approximately four days off training may be most beneficial for optimizing strength performance. In their initial study, Weiss et al. (27) had 54 untrained participants train their plantar flexors for eight weeks before testing 1RM heel raise and maximal low velocity isokinetic torque of the plantar flexors following short term training cessation. The only groups showing positive ES (enhanced performance) larger than trivial were three and four days of training cessation which showed small ES ( 0.30 and 0.38 , respectively). Their follow up study (28) involved 25 strength trained participants who trained for four weeks prior to testing 1 RM bench press and maximal low velocity isokinetic force of the bench press following short term training cessation. Only one absolute strength measure in one condition, maximal low velocity isokinetic force of the bench press at four days of training cessation, showed a greater ES than trivial, with a small ES of 0.26 . These results show some harmony between previous research and the current elite participants' trial and error-based practices, that three to four days of strength training cessation may be optimal for maximal strength expression.

Nearly all participants (90.9\%) removed accessory - or assistance, i.e. non-competition - exercises during the taper period, around two weeks out from an event. This serves two roles; firstly it reduces a large portion of 
training volume (participants in the current study lowered their training volume by nearly $60 \%$ ) which will allow for recovery from fatigue as part of the taper, and secondly, it acts to ensure training during the tapering period is competition focused and specific. Drastically reducing training volume during the taper has been shown to be an appropriate strategy to enhance recovery and to optimize performance across a range of sports $(14,19,29)$. The major goal in tapering for the overwhelming majority of participants $(90.9 \%)$ was to recover; this aligns with thoughts of previous researchers who have stated that the tapers primary aim is "to allow for physiological and psychological recovery from accumulated training stress" (16). Although training must be reduced to allow for recovery, some training should occur during the taper to avoid a loss of strength $(5,9,14)$. The need for an appropriate level of training during the taper was also reinforced in the current study as many participants (54.5\%) felt that poor tapering occurred when too much time was taken off from training. The training performed during tapering by our participants was on exercises that occur on competition day, so it is very specific, and this follows periodization principles which recommend specific training of lower volume near competition (18). If this same strategy is to be applied by athletes in other strength-related sports this would suggest that these athletes may benefit from performing strength exercises which directly assist aspects of sports performance when lowering training volume during the taper.

An interesting finding, that confirmed anecdotal reports, was that elite powerlifters require longer to recover from the deadlift than the other two competition lifts. Our findings showed that $90.9 \%$ of the elite powerlifters interviewed said the deadlift took longer to recover from and they also had their final deadlift session further out from competition than their final bench press and squat sessions to gain additional recovery time. However, no research was available that demonstrates it takes longer to recover from the deadlift than other compound movements. Interestingly, several elite powerlifters believed it was helpful to keep the bench press heavier prior to competition. Perhaps this additional recovery time for the deadlift is due to the absolute load of the deadlift being heavier, and so applying a greater stress to the body - especially the lower back (1), or a greater reliance on larger lower body muscle groups. However, no evidence is currently available to support these hypotheses. These findings require more research, but it may be prudent that athletes be cautious in programming the deadlift near competitions. 
Another novel finding extending current knowledge was that trial and error was involved in informing most participants' tapering strategy; that is learning how to taper from making mistakes or through past experiences of tapering. Such an approach could be enhanced through the use of a training log or diary to monitor athletes training and responses to tapering (4). Training diaries would allow athletes to more accurately reflect upon their tapering practices following competitions and make changes when strategies do not work, as well as replicate aspects that have successful results. Many participants interviewed in the current study used diaries to record their training information and utilized these when providing us with their training and tapering data. Reflection in athletes has been shown to occur more commonly amongst top performers (12); this may explain why some of the elite lifters $(63.6 \%)$ in the current study had an ability to change training during the taper based on 'feel', as they have reflected on previous experiences of how their body responded during tapering. Recommended tapering strategies can act as a guide, however, because individuals may have differing responses to strategies or come from different training backgrounds that could influence these responses (14), reflection will also allow individual's responses to be determined and implemented in future tapers. This strategy could also be implemented with other strength sports athletes; they could trial recommended strategies and use reflective practices to determine which methods were useful in improving performance following the taper. The large number of athletes utilizing trial and error as a learning strategy reinforces the need for further research to guide athletes in optimal tapering strategies (i.e. changes to training intensity, volume, frequency etc.) for maximal strength performance, the best ways to reflect on such training, as well as how to use science to communicate to specific populations.

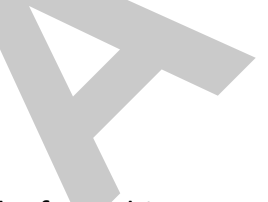

The results from this research may assist strength athletes in planning their taper, however, some limitations exist within the present research. Only elite raw New Zealand powerlifters were recruited in the current study, so different strategies may potentially be seen in lifters from other nations or from those who compete at a different level. Results may apply directly to powerlifting, a pure strength sport, but must be interpreted and modified to suit other sports where strength may not be the sole focus. 


\section{PRACTICAL APPLICATIONS}

Although the results of this study are specific to tapering for powerlifting, several of the findings have implications for other sports that benefit from strength training. For strength sports it would seem that athletes should not completely remove strength training prior to an important event, instead they should reduce the volume of strength training while keeping the intensity relatively high. Strength and conditioning coaches need to be aware that if too long of a time period is taken off strength training then force expression, and therefore performance, may decrease. If strength is to be optimized, specific to the sporting event, coaches should remove non-specific strength training exercises during the taper. Strength and conditioning coaches should prescribe only primary movements that directly assist sports performance in the strength program near an event, to assist with reduction in fatigue while maintaining/improving strength expression and performance. However, practitioners need to be careful to avoid going too heavy too often (over exertion or testing strength) prior to a competition as this may impair competitive performance. Coaches should potentially avoid deadlifting maximally close to important events as this may have some negative effect on recovery. Finally, coaches and athletes could make use of training diaries to more accurately track their sessions and changes in training load over the course of a training program. Such information can be used as the basis of reflective practice to help competitors optimize their tapering routines. 


\section{REFERENCES}

1. Cholewicki J, McGill S, and Norman R. Lumbar spine loads during the lifting of extremely heavy weights. Medicine and Science in Sports and Exercise 23: 1179, 1991.

2. Creswell JW and Miller DL. Determining validity in qualitative inquiry. Theory Into Practice 39: 124$130,2000$.

3. Flanagan EP. The Effect Size Statistic-Applications for the Strength and Conditioning Coach. Strength \& Conditioning Journal 35: 37-40, 2013.

4. Fleck SJ. Periodized strength training: a critical review. The Journal of Strength \& Conditioning Research 13: 82-89, 1999.

5. Gibala MJ, MacDougall JD, and Sale DG. The effects of tapering on strength performance in trained athletes. International Journal of Sports Medicine 15: 492-497, 1994.

6. Hakkinen K, Kallinen M, Komi PV, and Kauhanen H. Neuromuscular adaptations during short-term "normal" and reduced training periods in strength athletes. Electromyography and Clinical Neurophysiology 31: 35-42, 1991.

7. Hooper SL, Mackinnon LT, and Ginn EM. Effects of three tapering techniques on the performance, forces and psychometric measures of competitive swimmers. European Journal of Applied Physiology and Occupational Physiology 78: 258-263, 1998.

8. Hopkins WG. A scale of magnitudes for effect statistics. A New View of Statistics, 2002.

9. Hortobagyi T, Houmard JA, Stevenson JR, Fraser DD, Johns RA, and Israel RG. The effects of detraining on power athletes. Medicine and Science in Sports and Exercise 25: 929-935, 1993.

10. IPF. Technical Rules Book 2015. Luxemburg, 2014.

11. Izquierdo M, Ibanez J, Gonzalez-Badillo JJ, Ratamess NA, Kraemer WJ, Hakkinen K, Bonnabau H, Granados C, French DN, and Gorostiaga EM. Detraining and tapering effects on hormonal responses and strength performance. The Journal of Strength \& Conditioning Research 21: 768-775, 2007.

12. Jonker L, Elferink-Gemser MT, and Visscher C. Differences in self-regulatory skills among talented athletes: The significance of competitive level and type of sport. Journal of Sports Sciences 28: 901908, 2010. 
13. Le Meur Y, Hausswirth C, and Mujika I. Tapering for competition: A review. Science \& Sports 27: 77-87, 2012.

14. Mujika I. Intense training: the key to optimal performance before and during the taper. Scandinavian Journal of Medicine \& Science in Sports 20: 24-31, 2010.

15. Mujika I and Padilla S. Detraining: loss of training-induced physiological and performance adaptations. Part I. Sports Medicine 30: 79-87, 2000.

16. Mujika I, Padilla S, Pyne D, and Busso T. Physiological changes associated with the pre-event taper in athletes. Sports Medicine 34: 891-927, 2004.

17. Patton MQ. Qualitative research \& evaluation methods. Thousand Oaks, CA, USA: SAGE Publications, 2005.

18. Plisk SS and Stone MH. Periodization strategies. Strength \& Conditioning Journal 25: 19-37, 2003.

19. Pritchard H, Keogh J, Barnes M, and McGuigan M. Effects and Mechanisms of Tapering in Maximizing Muscular Strength. Strength \& Conditioning Journal 37: 72-83, 2015.

20. Pyne DB, Mujika I, and Reilly T. Peaking for optimal performance: Research limitations and future directions. Journal of Sports Sciences 27: 195-202, 2009.

21. Schinke RJ, Bonhomme J, McGannon KR, and Cummings J. The internal adaptation processes of professional boxers during the Showtime Super Six Boxing Classic: A qualitative thematic analysis. Psychology of Sport and Exercise 13: 830-839, 2012.

22. Sturges JE and Hanrahan KJ. Comparing telephone and face-to-face qualitative interviewing: a research note. Qualitative Research 4: 107-118, 2004.

23. Swinton PA, Lloyd R, Agouris I, and Stewart A. Contemporary training practices in elite British powerlifters: survey results from an international competition. The Journal of Strength \& Conditioning Research 23: 380-384, 2009.

24. Thomas $L$ and Busso T. A theoretical study of taper characteristics to optimize performance. Medicine and Science in Sports and Exercise 37: 1615-1621, 2005.

25. Tod DA, Bond KA, and Lavallee D. Professional development themes in strength and conditioning coaches. The Journal of Strength \& Conditioning Research 26: 851-860, 2012. 
26. Wade SM, Pope ZC, and Simonson SR. How prepared are college freshmen athletes for the rigors of college strength and conditioning? A survey of college strength and conditioning coaches. The Journal of Strength \& Conditioning Research 28: 2746-2753, 2014.

27. Weiss LW, Coney HD, and Clark FC. Optimal post-training abstinence for maximal strength expression. Research in Sports Medicine 11: 145-155, 2003.

28. Weiss LW, Wood LE, Fry AC, Kreider RB, Relyea GE, Bullen DB, and Grindstaff PD. Strength/power augmentation subsequent to short-term training abstinence. The Journal of Strength \& Conditioning Research 18: 765-770, 2004.

29. Wilson JM and Wilson GJ. A practical approach to the taper. Strength \& Conditioning Journal 30: 1017, 2008.

30. Winwood PW, Hume PA, Cronin JB, and Keogh JW. Retrospective injury epidemiology of strongman athletes. The Journal of Strength \& Conditioning Research 28: 28-42, 2014.

31. Winwood PW, Keogh JW, and Harris NK. The strength and conditioning practices of strongman competitors. The Journal of Strength \& Conditioning Research 25: 3118-3128, 2011.

\section{ACKNOWLEDGEMENTS}

We would like to thank the athletes who volunteered their time to participate, without their cooperation and willingness to share their training practices with us this study could not have gone ahead. 


\section{FIGURE LEGENDS}

Figure 1 . Tapering events timeline

Copyright (C 2015 Wolters Kluwer Health, Inc. Unauthorized reproduction of this article is prohibited. 


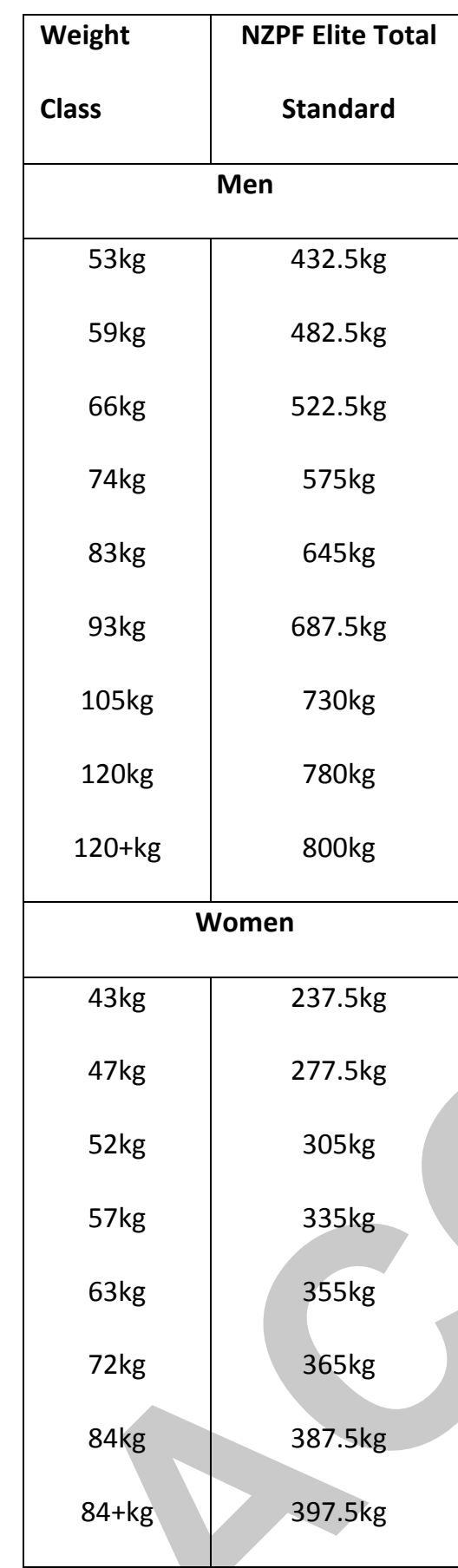

Table 1. NZPF elite total standards.

Copyright @ 2015 Wolters Kluwer Health, Inc. Unauthorized reproduction of this article is prohibited. 
1. When do you perform your final training sessions for each competition lift and what weights are used? Why?

2. When do you perform your final 'heavy' ( $>80 \% 1 \mathrm{RM})$ training sessions for each competition lift and what weights are used? Why?

3. Length of standard taper and why?

4. How does your volume, intensity and frequency change from pre to post taper?

5. How far out from an event do you begin to drop total training volume? And intensity? Does this differ according to the lift?

6. How do you determine to alter the loads, reps etc. as you do?

7. Do you remove your assistance exercises or training equipment (belts, wraps etc.)? If so, when and what do you remove? Why?

8. When tapering hasn't worked, what went wrong and why?

9. Is there any other information on your tapering or training practices that you think may be of interest?

Table 2. The questions the participants were asked during the interviews. 


\begin{tabular}{|c|c|c|}
\hline Final Training Session Type & Days Out from Competition & Top Set(s) Percentage of 1RM \\
\hline Final heavy squat session & $8.0 \pm 2.9 *$ & $90.0 \pm 5.4$ \\
\hline Final squat session & $4.0 \pm 1.8 *$ & $66.0 \pm 15.7$ \\
\hline Final heavy bench press session & $7.3 \pm 2.7 *$ & $92.2 \pm 5.7^{\#}$ \\
\hline Final bench press session & $4.0 \pm 1.8 *$ & $67.3 \pm 18.1$ \\
\hline Final heavy deadlift session & $10.9 \pm 4.0$ & $88.9 \pm 6.1$ \\
\hline Final deadlift session & $7.4 \pm 4.1$ & $72.6 \pm 18.5$ \\
\hline \multicolumn{3}{|c|}{${ }^{*}$ denotes significant difference compared to relevant deadlift session; ${ }^{\#}$ denotes significant difference } \\
\hline
\end{tabular}

Table 3. Final training sessions.

Copyright @ 2015 Wolters Kluwer Health, Inc. Unauthorized reproduction of this article is prohibited. 


\begin{tabular}{|c|c|}
\hline Theme & $\begin{array}{c}\text { Sample Representativeness } \\
\qquad * \text { (\% of sample) }\end{array}$ \\
\hline \multicolumn{2}{|l|}{ Tapering differences between lifts } \\
\hline More recovery needed for the deadlift & General (90.9\%) \\
\hline Bench press \& squat tapering performed in a similar manner & Variant $(45.5 \%)$ \\
\hline Bench press can stay heavier for longer & Variant (36.4\%) \\
\hline \multicolumn{2}{|l|}{ Information sources for taper strategy } \\
\hline Trial \& error & Typical (81.8\%) \\
\hline By 'feel' & $(63.6 \%)$ \\
\hline Previous coaches & Variant (36.4\%) \\
\hline Other lifters & Variant (18.2\%) \\
\hline \multicolumn{2}{|l|}{ Motives for tapering strategy } \\
\hline To fully recover & General (90.9\%) \\
\hline Light competition movements for familiarity & Variant $(45.5 \%)$ \\
\hline Time off heavy work makes them 'hungry' to lif & Variant (18.2\%) \\
\hline \multicolumn{2}{|l|}{ Taper focus is on the competition lifts } \\
\hline Accessory work is removed during the taper & General (90.9\%) \\
\hline Equipment is used on heavy sets as it would be in competition & Typical (81.8\%) \\
\hline \multicolumn{2}{|l|}{ Other strategies employed during the taper } \\
\hline Nutrition & Typical (72.7\%) \\
\hline Mobility Work - stretching, foam rolling, massage etc. & Variant (45.5\%) \\
\hline \multicolumn{2}{|l|}{ Poor tapering experiences } \\
\hline Taking too much time off -1 week+ & Typical (54.5\%) \\
\hline Over exertion too often prior to taper & Variant (36.4\%) \\
\hline Cut too much weight & Variant (36.4\%) \\
\hline \multicolumn{2}{|c|}{ General = themes applying to all or all but one participant; typical = themes applying to more than half the } \\
\hline \multicolumn{2}{|c|}{ participants, but less than general; variant = themes applying to two or more subjects, but less than typical. } \\
\hline Note: Participants could be included in more than one subtheme, henc & may add to more than $100 \%$ \\
\hline
\end{tabular}

Copyright @ 2015 Wolters Kluwer Health, Inc. Unauthorized reproduction of this article is prohibited. 
Table 4. Broad themes and subthemes from the interviews and the sample representativeness.

Copyright (C 2015 Wolters Kluwer Health, Inc. Unauthorized reproduction of this article is prohibited. 
$\underline{\text { Tapering Events Timeline }}$

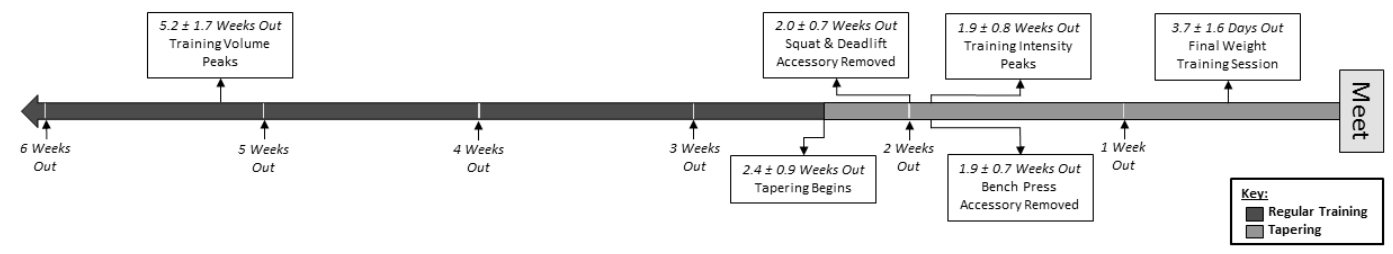

Copyright (C 2015 Wolters Kluwer Health, Inc. Unauthorized reproduction of this article is prohibited. 